\title{
Elucidating the effect of subsurface structure on mineral dissolution rates using reactive transport modeling
}

\author{
ELIZABETH ANDREWS ${ }^{1}$, JEFFREY HYMAN ${ }^{2}$, \\ MATTHEW SWEENEY ${ }^{2}$, DAVID MOULTON ${ }^{2}$ AND ALEXIS \\ NAVARRE-SITCHLER ${ }^{1}$ \\ ${ }^{1}$ Colorado School of Mines \\ ${ }^{2}$ Los Alamos National Laboratory \\ Presenting Author: eandrews1@mines.edu
}

Heterogeneity in natural systems plays a critical role in creating reaction hotspots, or zones of fast reaction, immediately adjacent to zones with minimal reaction. This is especially true in heterogeneous systems of fractured rock, where the fractures act as fast flowing conduits while reaction in the matrix blocks is often diffusion-limited. However, it is difficult to investigate these systems in numerical simulations because of the computational expense associated with simulating discrete fractures in a porous medium. Recent advances in coupled fracture network and reactive transport codes have provided the tools to investigate mineral-fluid reactions in fractured rock. Previous work has investigated the role of fracture density on mineral dissolution rates, and this work has demonstrated that the heterogeneity associated with fractures can reduce the mineral dissolution rate by several orders of magnitude. Here, we use site-inspired domains to investigate the importance of subsurface structure, relative to surface processes in determining mineral dissolution rates.

The Gordon Gulch catchment of the Boulder Creek Critical Zone Observatory has significant differences in fracture density and groundwater solute concentrations between north and south facing slopes. The groundwater solute difference has been attributed to differential rates of mineral dissolution between the two hillslopes. In this study, we use numerical simulations to investigate the role fractures play in water delivery to the subsurface and the impact of this on mineral dissolution rates. With the site inspired domains, we investigate the importance of fracture topology, relative to surface processes, including the magnitude and timing of precipitation in determining the rates of mineral dissolution, and solute generation on the hillslopes. 